\title{
Vascular structures and relationship to intramuscular fat in supraspinatus muscle following tendon detachment and reattachment - an experimental study in rabbits
}

\author{
M. Maclntyre-Newell1,2, H.K. Uhthoff', G. Trudel1,3, O. Laneuville ${ }^{1,2}$ \\ 1 Bone and Joint Research Laboratory, Faculty of Medicine, University of Ottawa, Ottawa, Canada \\ 2 Department of Biology, Faculty of Science, University of Ottawa, Ottawa, Canada \\ 3 Department of Medicine, Bone and Joint Research Laboratory, The Ottawa Hospital Rehabilitation Centre, \\ Ottawa, Canada
}

\section{CORRESPONDING AUTHOR:}

Odette Laneuville

Phone: 6135625800 ext. 4606

Fax: 6135625486

E-mail: olaneuvi@uottawa.ca

DOI:

10.32098/mltj.01.2019.10

\begin{abstract}
SUMMARY
Background. Supraspinatus (SSP) tendon tear leads to intramuscular fat accumulation and the mechanisms are unknown. We investigated changes in SSP muscle vascularization and association with intramuscular fat following SSP tendon detachment with or without reattachment. Methods. Sixty-six rabbits underwent SSP tendon detachment. In groups of ten, thirty rabbits were sacrificed 4, 8, and 12 weeks following detachment. In groups of twelve, the remaining thirty-six rabbits underwent SSP tendon reattachment 4, 8, and 12 weeks after detachment and were sacrificed 12 weeks later. Vascularization was quantified using CD31 immunohistochemistry and tested for correlation with intramuscular fat measurements. Results. Four weeks after SSP tendon detachment, vascularization increased in the distal SSP muscle, reaching significance after 12 weeks of detachment $(\mathrm{p}=0.024)$. Vascularization was correlated with intramuscular fat accumulation after detachment $(r=0.29 ; \mathrm{p}=0.008)$. No significant correlation was observed in the SSP tendon reattachment groups $(r=0.06 ; p=0.65)$. Some vascular structures in the reattachment group had thicker vascular walls. Conclusion. Increased vascularization in the distal SSP muscle was observed following tendon detachment but not after surgical reattachment. Both increased vascularization and intra-muscular fat accumulation co-localized to the distal end near the tendon tear site.
\end{abstract}

KEY WORDS

intramuscular fat; rotator cuff tear; vascularization; vascular wall thickness

\section{BACKGROUND}

Rotator cuff tears are a significant clinical problem that affects $35 \%$ of individuals over 60 years of age (1). Shoulder pain and reduced range of motion observed by physical examination often accompany the rotator cuff tear (1). In addition to shoulder examination, imaging provides information about muscle retraction, atrophy, and intraand extramuscular fat accumulation. The muscle changes compound shoulder function and contribute to low surgical success rates (2-4). Rotator cuff tears have also been associated with thyroid disease impacting metabolic functions of the tendon (5). Fat accumulation is a criterion frequently used as a prognostic factor to determine a patients' eligibility for surgical repair $(2,4,6)$. Using magnetic resonance imaging (MRI), fat accumulation is scored on the Goutallier scale, a semi-quantitative assessment used to guide decisions for surgical intervention $(3,4,6)$. Intramuscular fat accumulation in rotator cuff tears has been shown to have a strong association with a negative surgical outcomes (3). Despite its important clinical relevance, little is known about the origin of intramuscular fat depots and the cellular processes precipitating fat accumulation.

The rabbit model of rotator cuff tear has provided insight into fat accumulation observed in the supraspinatus (SSP) muscle 
after a complete detachment performed at the humeral insertion. Over time, intramuscular fat accumulation is localized at the distal end of the muscle near the tendon tear, accumulates on an increasing distal-to-proximal gradient, and persists following surgical tendon reattachment (7-12). The muscular adipose tissue accumulation is characterized by the presence of both intra and extra-muscular fat $(7-10,13,14)$. Extramuscular adipose tissue surrounds skeletal muscle, while intramuscular adipose tissue is found between individual muscle fascicles within a skeletal muscle (13). Liu et al. have recently found that a matrix metalloproteinase- 13 knockout will exacerbate fat accumulation following a complete rotator cuff tear (15). Intramuscular fat accumulation has been observed with ageing and also in individuals with insulin resistance, metabolic, orthopaedic and neurologic conditions (16-18). High levels of intramuscular fat were associated with a loss of muscle strength and reduced mobility $(13,16)$.

In other models of fat accumulation such as subcutaneous fat depots, vascularization has been associated with the development and proliferation of adipocytes $(18,19)$. Adipose tissue is reliant on vascularization for growth and proliferation; both processes are associated with a higher demand for oxygen and circulating fatty acids, and endocrine function (19). The suprascapular artery principally supplies the SSP muscle; it enters through the midsection of the muscle belly and diverges toward both ends, distal and proximal (20-22). The diameter of vascular structures decrease from the entry point to the proximal and distal ends $(20,22)$.

The purpose of this study is to provide insight into the pathophysiology of intramuscular fat accumulation in the SSP muscle by correlating it with vascularization following a complete SSP tendon detachment, with or without surgical reattachment. We first hypothesized that following SSP tendon detachment there would be 1) an increase in SSP muscle vascularization; 2) a positive correlation between vascularization and intramuscular fat accumulation in SSP muscle; 3) surgical reattachment would prevent both vascularization increase and fat accumulation.

\section{MATERIALS AND METHODS}

After obtaining institutional animal care committee approval (Protocol Number ME-2479), ninety-nine female New Zealand white rabbits $(3.0 \mathrm{~kg})$ were randomly assigned into two experimental groups: SSP tendon detachment (detachment) and SSP tendon reattachment (reattachment). All experiments were carried out in accordance with outlined ethical standards (23).

\section{Detachment group}

Thirty rabbits underwent SSP detachment at the distal end near the footprint, alternating left and right shoulders.
The rabbits were sacrificed in groups of ten 4,8 , and 12 weeks after detachment. The remaining fifteen rabbits had no surgical intervention and both shoulders were harvested in groups of five at the same time points to serve as age-matched controls.

The details of the surgical intervention to detach the SSP tendon have been previously published (7-9). In short, SSP tendons were transected from the insertion at the greater tuberosity under general anesthesia. The distal stump of the tendon was wrapped with a polyvinylidene fluoride membrane to prevent adhesion. Fentanyl and buprenorphine were administered for 3 days post-operative to control pain. Rabbits were housed individually, provided free access to food and water, and were not immobilized.

\section{Reattachment group}

Thirty-six rabbits underwent SSP tendon detachment, the surgical procedure is described above, at the distal end near the footprint, and were reattached in groups of twelve 4, 8 , and 12 weeks after detachment. The details of the surgical intervention to perform tendon reattachment have been previously published (9). Briefly, through the original skin incision the polyvinylidene fluoride membrane on the SSP tendon was removed. Surgery was performed to create a bony trough at the medial wall of the greater tuberosity. Three $1 \mathrm{~mm}$ holes were drilled into the bony trough. Using two nonabsorbable 3-0 Prolene sutures, a modified Mason-Allen technique was used to complete the transosseus reattachment $(8,24)$. A period of twelve weeks healing following the SSP tendon reattachment was allowed before the shoulders were harvested. Eighteen rabbits did not undergo any surgical intervention and both shoulders were harvested in groups of six at the same time points to serve as age-matched controls.

\section{Collection of specimens}

All rabbits were sacrificed using a pentobarbital overdose and shoulders were harvested en bloc avoiding dissection of the $\mathrm{SSP}$ muscle from the scapula. Specimens were frozen at $-20^{\circ} \mathrm{C}$ until processing. The time points are identified as number of weeks following detachment + number of weeks after surgical reattachment: $4,8,12,4+12,8+12$, and $12+12$ weeks.

\section{Tissue preparation}

Following formalin fixation, $1 \mathrm{~mm}$ sagittal sections were cut from the proximal, middle, and distal regions of the SSP muscle and processed to paraffin. $5 \mu \mathrm{m}$ thick sections were cut from each paraffin bloc and mounted for immunohistochemistry.

Muscles, Ligaments and Tendons Journal 2019;9 (1) 
Immunohistochemistry: staining and analysis of vascular structures

A commercially available and pre-diluted CD31 antibody (131M-98, Cell Marque, Rocklin, CA, USA) was used to stain the endothelial layer of vascular structures. Deparaffinized sections underwent heat and pressure induced epitope retrieval in a sodium citrate buffer $(\mathrm{pH} 9)$ at $110{ }^{\circ} \mathrm{C}$ in a pressurized environment for 13 minutes, using an AR Histo S5 Rapid Microwave Histoprocessor (PALM Histology Core Facility, University of Ottawa). The sections were then incubated with the primary CD31 antibody for one hour at room temperature. The secondary goat-anti-mouse IgG antibody (ab6789, Abcam, Cambridge, UK) diluted at 1:200 was added for one hour at room temperature. The sections were then developed using 3, 3'-diaminobenzidine (BDB2004L, Biocare Medical, Pacheco, CA, USA) for 1-3 minutes, counterstained with hematoxylin, and mounted for analysis. The CD31 stained sections were then scanned using an Aperio CS2 ScanScope (Leica Biosystems, Vista, CA, USA), and whole SSP muscle cross-sections were digitized. From each digital SSP muscle cross-section, seven fields of view (FOV) measuring $0.855 \mathrm{~mm} \times 0.530 \mathrm{~mm}$ $\left(0.453 \mathrm{~mm}^{2}\right)$ were randomly placed at $1 \mathrm{x}$ magnification, and vascular structures were manually counted at a higher magnification. The vascular structures are defined as all structures with positive CD31 staining, one or more stained endothelial nuclei, and located within the intramuscular space. Thick vascular structures were distinguished from the total number of vascular structures if they met the following criteria: positive CD31 staining, vascular wall thickness of greater than $15 \mu \mathrm{m}$, and diameter greater than $50 \mu \mathrm{m}$.

\section{Intramuscular fat staining and analysis}

Intramuscular fat accumulation from the same specimens has been previously measured on osmium tetroxide fixed sections at the same sites as the current study and the data published $(8,9)$.

\section{Data and statistical analysis}

The number of vascular structures from 7 FOVs per muscle section are reported as median \pm standard deviation. The number of thick vascular structures was expressed as (number of thick vascular structures / total number of vascular structures) x $100(\%)$. ANOVA (one-way) and unpaired t-tests were used to compare experimental groups with age-matched controls. Pearson correlations were used to determine if a significant correlation existed between the number of vascular structures and intramuscular fat accumulation. In the Pearson correlation analysis, both vascu- lar structure data and intramuscular fat data take into consideration total SSP muscle cross-sectional area: median number of vascular structures from $7 \mathrm{FOV} /$ total SSP muscle cross-sectional area; total intramuscular fat area/SSP muscle cross-sectional area (\%). A p-value of less than 0.05 indicated statistical significance, and all data was analyzed in the $\mathrm{R}$ environment (Version 1.0.153).

\section{RESULTS}

\section{Vascular structures}

Images of SSP muscles with CD31 stained vascular structures in detached and control groups are shown in figures $\mathbf{1}$ and $\mathbf{2}$, panels a to $\mathbf{f}$. Twelve weeks after detachment, there were significantly more vascular structures in the distal SSP muscle $(4.78 \pm 2.49$ vs $2.44 \pm 0.088$ vascular structures; $\mathrm{p}$ $=0.024$ ) when compared to control SSP muscles, but not at four and eight weeks after detachment (figure 1g). There was no statistically significant difference between the 3 muscle regions studied. After SSP tendon reattachment, the number of vascular structures in SSP muscles was comparable to control SSP muscles at all time points and in distal, middle, and proximal regions of the SSP muscle (figure 2, panel $\mathbf{g}$ ).

Observation of thick vascular structures was defined by positive CD31 staining, vascular wall thickness greater than $15 \mu \mathrm{m}$, and diameter greater than $50 \mu \mathrm{m}$. There was a trend for a higher mean percentage of thick vascular structures in SSP muscles after tendon reattachment in seven of the nine groups, time-points and locations, when compared to controls (figure 2, panels a, b, c, and $\mathbf{d}$ and table I). Statistical significance was reached only in the $4+12$ weeks group in the distal region of the SSP muscle $(\mathrm{p}=0.025$, table $\mathbf{I})$.

\section{Correlations between vascular structures and intramuscular fat}

The intramuscular fat was more prominent in the distal portion of the SSP muscle 12 weeks after detachment. SSP tendon reattachment did not reverse SSP muscle intramuscular fat accumulation and fat staining remained elevated in the distal section of the SSP muscle after reattachment (figure 3). The number of vascular structures was positively correlated with intramuscular fat accumulation in detached SSP muscles $(r=0.29, p=0.008)$. No significant correlation existed in the control group $(r=-0.1986, p=0.11$; figure 4; table II). After reattachment of the SSP tendon, no correlation was detected between vascularization and intramuscular fat in both the reattachment and the control groups (figure 5; table II). 

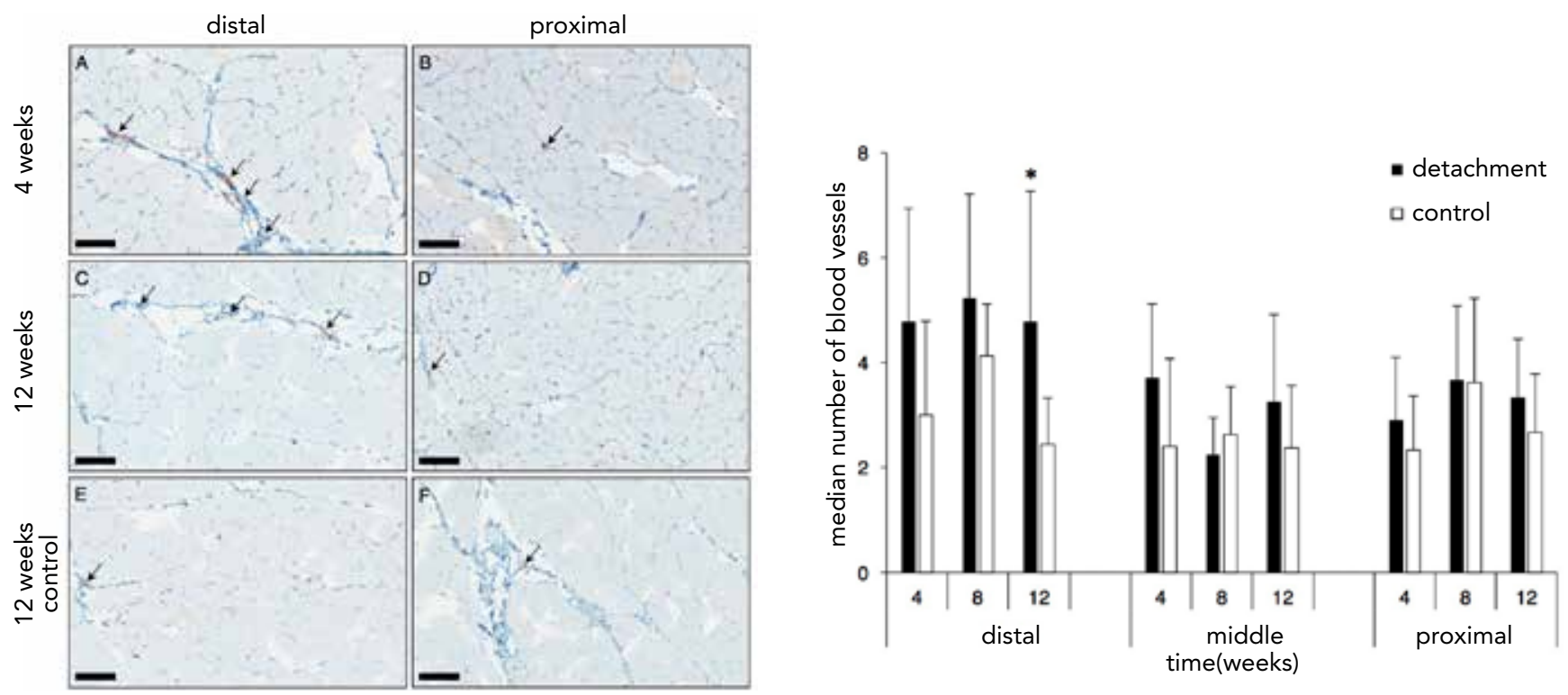

Figure 1 - SSP muscle after SSP tendon detachment. Panels a-f: micrographs of entire FOV from sagittal section of the SSP muscle at distal and proximal regions, 4 and 12 weeks after SSP tendon detachment. Vascular structures (black arrows) were stained with a CD31 antibody (brown) and counterstained with hematoxylin (blue). Panel g shows median number of vascular structures \pm one standard deviation. More vascular structures were found in the distal SSP muscle 12 weeks after SSP tendon detachment when compared to age-matched controls $(p=0.024)$. Distal SSP muscle sections ( $\mathbf{a}$, $\mathbf{c})$ showed a trend towards more vascular structures at 4 weeks after detachment compared to proximal sections (b, $\mathbf{d}$ ) of the same SSP muscle and compared to sections from the control groups $(\mathbf{e}, \mathbf{f})$. Original magnification is $20 x$. Scale bars indicate $100 \mu m$. ${ }^{\star} p<0.05$.
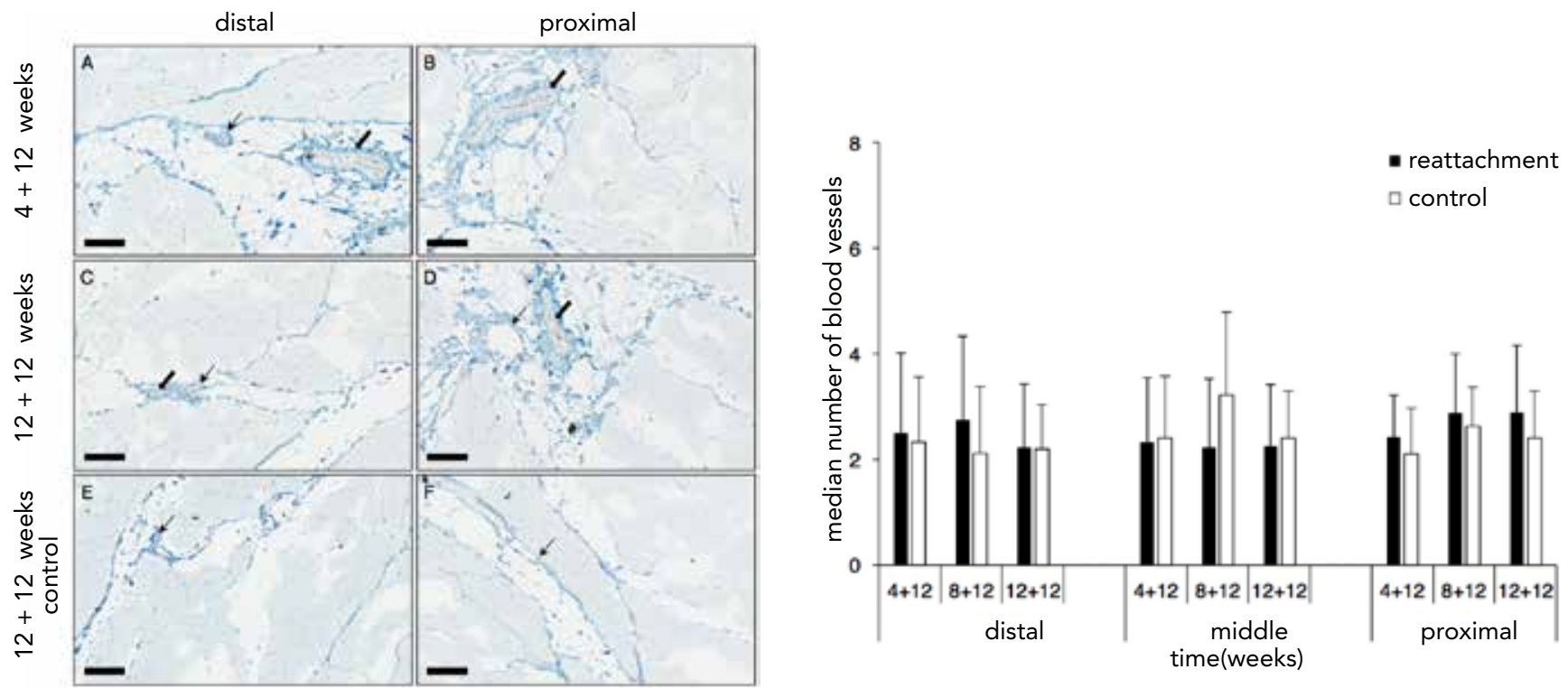

Figure 2 - SSP muscles 12 weeks after surgical reattachment. Micrographs of entire FOV of sagittal sections of distal and proximal regions of the SSP muscle reattachment group at $4+12(\mathbf{a}, \mathbf{b})$ and $12+12$ weeks $(\mathbf{c}, \mathbf{d})$ and age-matched controls (e, $\mathbf{f})$. Vascular structures stained with a CD31 antibody (brown and indicated with arrows) and myocytes stained with hematoxylin (blue). Some vascular structures with thick walls (indicated with thick arrows) were observed in the reattachment groups (a, b, $\mathbf{c}$, d) but not in controls $(\mathbf{e}, \mathbf{f})$. Original magnification is $20 x$. Scale bars indicate $100 \mu \mathrm{m}$. Panel g shows median number of vascular structures for each group \pm one standard deviation. There was no statistically significant difference between groups. 
Table I - Percentage of vascular structures with thick vascular walls in the detachment and reattachment SSP muscles for all time points. Vascular structures were counted as thick when they met the following criteria: positive CD31 staining, greater than 15 $\mu \mathrm{m}$ vascular wall thickness, and a diameter of $50 \mu \mathrm{m}$ or larger. The number of thick vascular structures / total number of vascular structures for each FOV are presented.

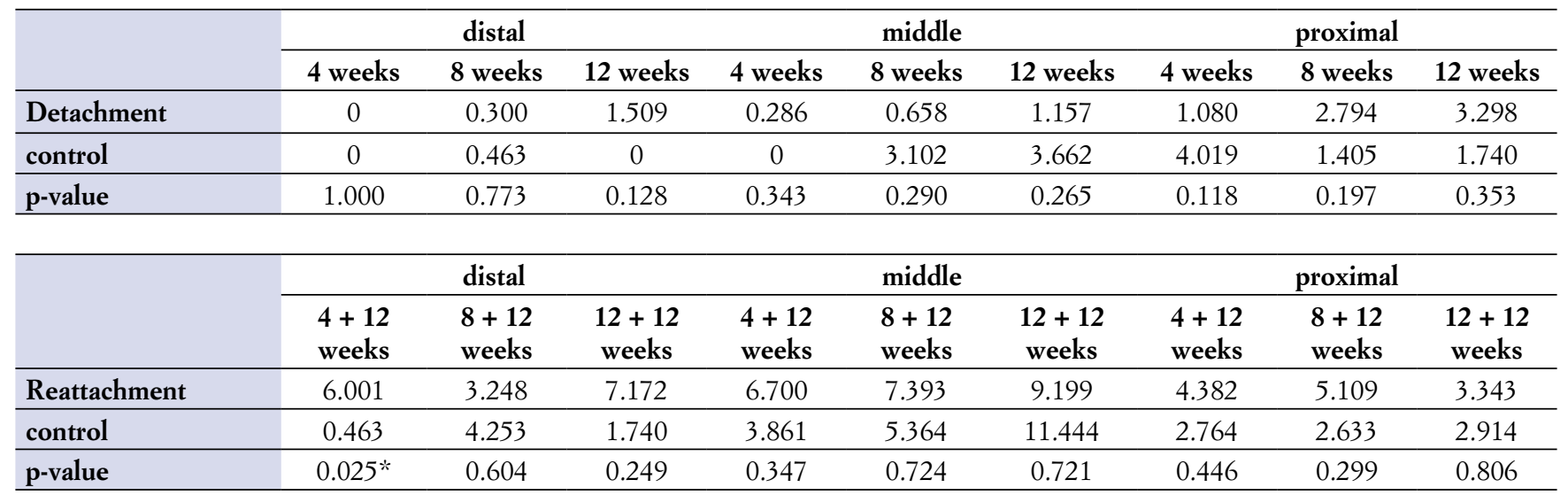

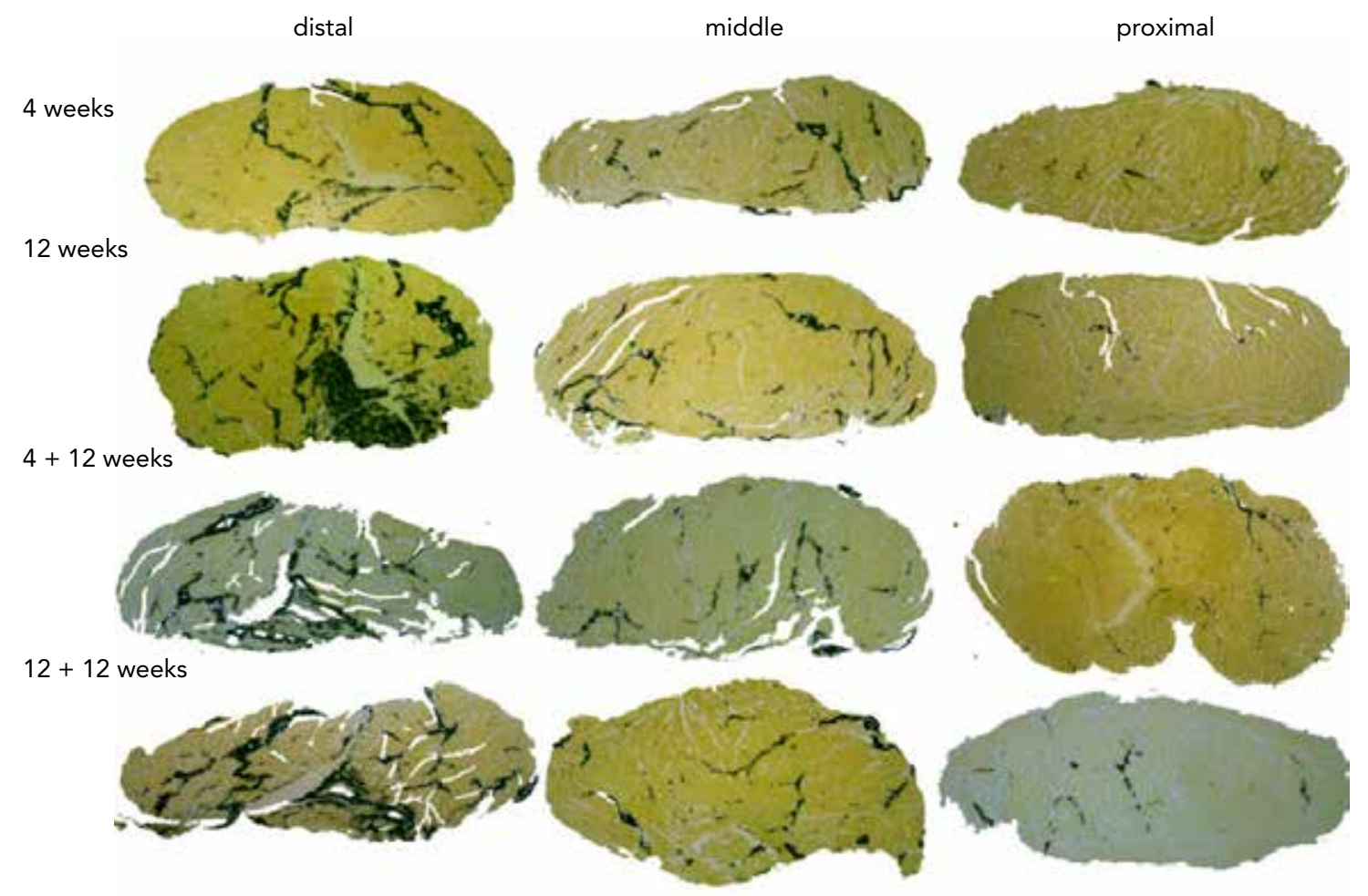

Figure 3 - Micrographs of entire SSP muscle cross sections fixed with osmium tetroxide staining intramuscular fat black. Fat accumulation was more pronounced in the distal rather than the proximal section of the SSP muscle after detachment. The progressive fat accumulation after detachment was not reversed by surgical reattachment. Control specimens showed minimal basal intramuscular fat accumulation. Original magnification is $6.7 \mathrm{x}$. 


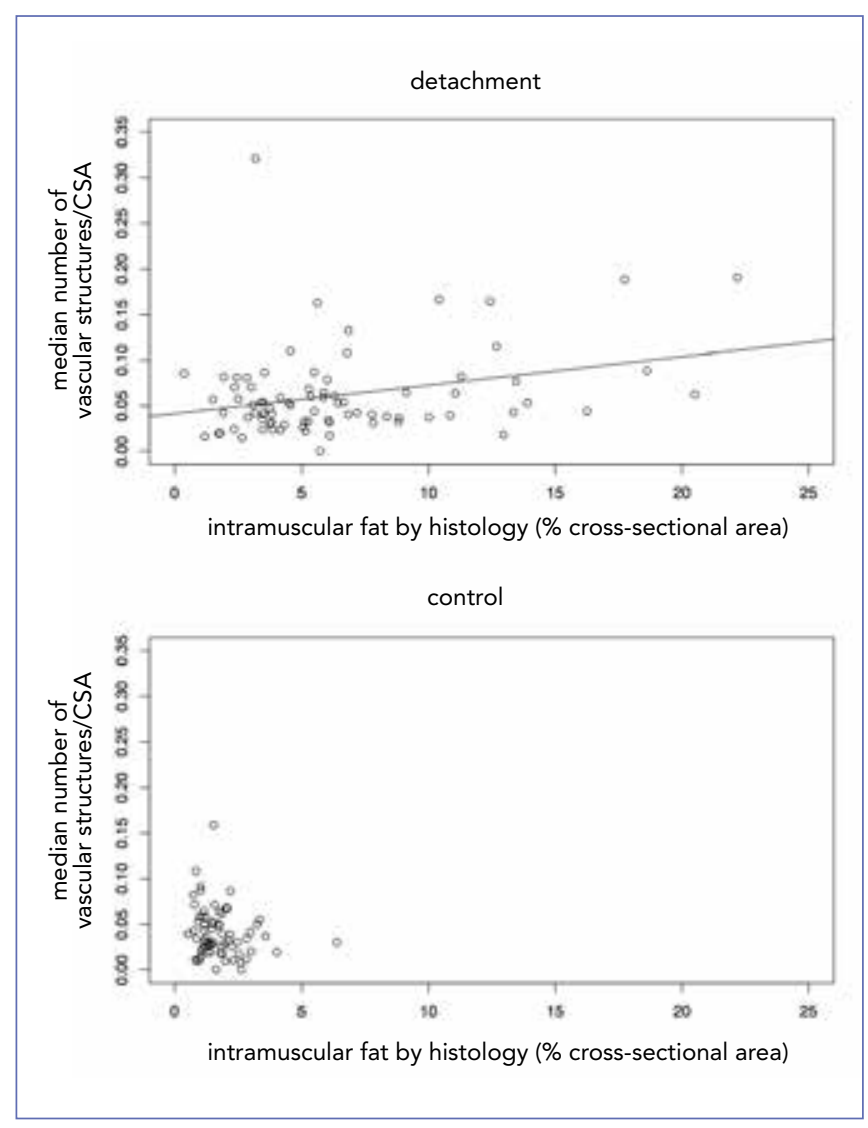

Figure 4 - Correlation graphs of vascular structures and corresponding intramuscular fat accumulation following SSP tendon detachment. Detachment and control values have been pooled over time (4, 8, 12 weeks) and SSP muscle location (distal, middle, proximal). The Pearson correlation coefficient was significant for the detachment group $(0.2935, p=$ $0.0078)$. No significant correlation was found in the control group (-0.1986, $p=0.1072)$.

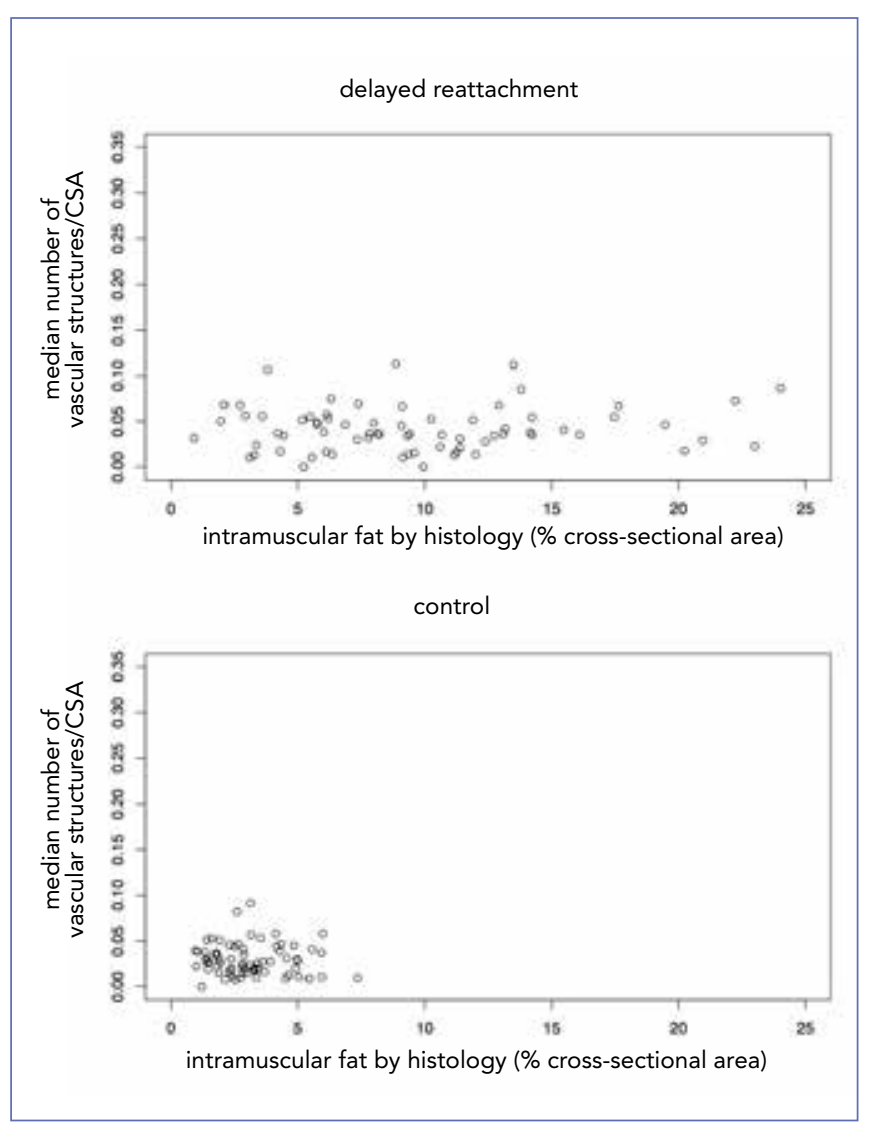

Figure 5 - Correlation between vascularization and intramuscular fat after SSP tendon detachment and surgical reattachment and age-matched controls. Data from distal, middle, and proximal regions, and $4+12,8+12$, and $12+12$ durations were pooled for each respective graph. There was no significant correlation between vascular density and intramuscular fat 12 weeks after SSP tendon reattachment.

Table II - Pearson correlation coefficients and exact p-values for all comparisons of vascular structure numbers and corresponding intramuscular fat content from all groups. Pearson correlation coefficients, exact p-value, and the sample size for each group are indicated and comparisons are reported.

\begin{tabular}{llccc}
\hline Comparison & & Pearson correlation coefficient & p-value & n \\
Detachment group & control & -0.1986 & 0.107 & 67 \\
\cline { 2 - 5 } & experimental & 0.2935 & $0.008^{*}$ & 84 \\
\cline { 2 - 5 } & experimental distal & 0.6496 & 0.092 & 27 \\
\cline { 2 - 5 } & experimental middle & 0.3762 & 0.058 & 26 \\
\cline { 2 - 5 } & experimental proximal & 0.0160 & 0.936 & 28 \\
\hline Reattachment group & Control & -0.0527 & 0.665 & 70 \\
\cline { 2 - 5 } & Experimental & 0.0552 & 0.645 & 72 \\
\hline
\end{tabular}




\section{DISCUSSION}

The main finding of our study was increased vascularization in the distal portion of the SSP muscle reaching statistical significance following 12 weeks of tendon detachment only. Twelve weeks after reattachment of the SSP tendon, the number of vascular structures in SSP muscles returned to control levels at all 3 muscle locations studied, irrespective of the delay before reattachment. The data indicates the dynamic nature of muscle vascularization in response to SSP tendon detachment and reattachment. Furthermore, the vascularization correlated with intramuscular fat accumulation in the distal portion of the detached SSP muscles. The increased vascularity of the distal SSP muscle and the correlations with intramuscular fat accumulation were no longer present 12 weeks following tendon reattachment.

The animal model used in this study allows histological access to tissue for evaluation at different time points with different interventions. This model imperfectly represents clinical rotator cuff disease; rabbits are quadrupeds and tendons were not degenerated at detachment procedure. Complete detachment of the SSP tendon was studied and the conclusions may not apply to partial tears. The observations made in this study are limited to 12 weeks following tendon detachment, and 12 weeks after surgical reattachment; longer follow up times remain to be studied. The sample size was large (112 rabbits), but multiple conditions of intervention, duration, and control groups reduced statistical power to assess vascularization. Overall, the methods used in this study allowed for access to tissue to gain an understanding of the changes in vascularization following rotator cuff tear, but further studies need to be conducted to relate these findings to the clinical presentation of rotator cuff tears.

The vascular response to SSP tendon detachment in eighteen human cadavers has been previously studied, and provided evidence of increased vascularity on the bursal side of the tendon (25). The SSP tendon response to injury is likely to involve the suprascapular artery, the main blood vessel contributing to the blood supply of the distal SSP tendon. Increased vascular density in SSP tendon was confirmed in other animal models following SSP tendon detachment $(26,27)$. The response of vascularization to tendon damage is indicative of the tendon's ability to repair, by allowing cell infiltration from the circulatory system and the necessary reparative factors, including those participating in the inflammatory response (28). In the patellar tendon, neovascularization was associated with increased pain during loading of the knee joint (29). In the context of human rotator cuff tears, Lakemeir et al. showed that increased vascular density can accelerate tendon degeneration by weakening the functional structure of the tendon
(30). Overall, increased vascularization of tendons has been shown to associate with pain and weakening of the tendon, while being required for cell proliferation and healing. Our finding of increased vascularization in the SSP muscle adds to previous observations on the pathophysiology of rotator cuff tears and provides evidence for localized increase in blood vessel density occurring in the distal portion of the SSP muscle, where intramuscular fat accumulates near the site of tendon tear.

Changes in SSP muscle vascularization were tested for correlations with the intramuscular fat accumulation using data previously published from the same specimens $(8,9)$. Increased vascularization of the SSP muscle was correlated with intramuscular fat accumulation. This correlation was not observed in the reattachment group despite significant intramuscular fat accumulation, while the number of vascular structures were comparable to control values.

The cellular and molecular mechanisms leading to intramuscular fat accumulation after rotator cuff tear have not been elucidated. In the current study, we measured increased vascular density and proposed a potential contribution to fat accumulation in the SSP muscle. Other potential mechanisms contributing to intramuscular fat accumulation have been described in the literature. Previous studies have demonstrated fibro-adipogenic progenitor cells resident in skeletal muscles contribute to intramuscular fat accumulation following rotator cuff tears in a mouse model (31). The mechanism by which vascularization could contribute to intramuscular fat accumulation may involve pericytes, the contractile cells in vascular structures that are progenitors for myocytes and adipocytes (32). The mechanism proposed by these authors included an accumulation of pericytes in the endothelial space of developing vascular structures with the ability to differentiate into adipocytes and myocytes following muscle injury $(19,32,33)$. Another mechanism by which newly formed muscle vascularization could contribute to fat accumulation was proposed by Medici et al. (34). These authors identified cells, originating from vascular endothelium and that can be converted into multipotent stem-like cells, progenitors for adipocytes (34). The relationship between vascularization and intramuscular fat we report is consistent with this mechanism. The sources of adipocytes contributing to increased fat accumulation after tendon tear remains to be determined, including the potential contribution of mesenchymal stem cells (MSCs) residing in muscles with the capacity to differentiate into adipocytes upon muscle damage.

Vascular changes were not limited to increased vascular density; vascular structures with increased thickness of the sub-endothelial layer were observed in the reattachment group. Vascular thickening has previously been described 
in association with hypertension and ageing but little is known about consequences on muscle physiology (37). It has also been reported that increased vascular thickness can decrease vascular compliance, preventing proper secretory function of vascularization, potentially impacting fat accumulation in the muscle $(37,38)$. Pericytes, progenitors for adipocytes or skeletal myocytes, can accumulate in the sub-endothelial space and contribute to increased vascular thickness and intramuscular fat accumulation (19,32,33). In the current animal model of rotator cuff tear, acute inflammation, induced by surgical detachment, could be responsible for the initial increase in vascularization while chronic inflammation, persisting after surgical reattachment, could be responsible for the increased vascular thickness. Our observation of increased vascular wall thickness in association with the surgical reattachment of the SSP tendon deserves further investigation, including a potential role in fat accumulation persisting after repair.

Interestingly, surgical reattachment performed after 4,8 , or 12 weeks of tendon detachment did not change the number of vascular structures in the SSP muscle measured at 12 weeks after reattachment. The lack of change in vascular density in reattached specimens could have been caused by the restoration of tendon continuity and tensile strength of the SSP muscle, therefore terminating the stimuli for neovascularization. The persistence of intramuscular fat observed in reattachment groups is likely to involve mechanisms other than vascular changes. Gregory et al. have shown that adipocytes cultured in vitro can develop in skeletal muscle cells originating from non-hematopoietic MSCs of the bone marrow, and in the absence of vascularization (40). Different precursor cells for skeletal muscle adipocytes have been identified and, in some cases, their differentiation does not require vascularization (40). The cellular sources of skeletal muscle adipocytes accumulating in SSP muscle following SSP tendon detachment remains to be established.

\section{REFERENCES}

1. Yamamoto A, Takagishi K, Osawa T, Yanagawa T, Nakajima D, Shitara H, et al. Prevalence and risk factors of a rotator cuff tear in the general population. J Shoulder Elb Surg [Internet] 2010; 19(1):116-120. Available from: http://dx.doi. org/10.1016/j.jse.2009.04.006

2. Nardo L, Karampinos DC, Lansdown DA, Carballido-Gamio J, Lee S, Maroldi R, et al. Quantitative assessment of fat infiltration in the rotator cuff muscles using water-fat MRI. J Magn Reson Imaging 2014; 39(5):1178-1185.

3. Giambini H, Hatta T, Krzysztof GR, Widholm P, Karlsson A, Leinhard OD, et al. Intramuscular fat infiltration evaluated by magnetic resonance imaging predicts the extensibility of the supraspinatus muscle. Muscle and Nerve 2017; 1-7.
This study investigated the number of blood vessels in the SSP muscle using a rabbit model of rotator cuff tear and repair. Histology and CD31 immunohistochemistry analyses were used to quantitate blood vessel numbers in the distal, middle, and proximal regions of the detached SSP muscle, and results were compared with intramuscular fat measures from the same specimens. Four weeks after SSP tendon detachment, vascularization increased in the distal SSP muscle reaching significance after 12 weeks of tendon detachment and was correlated with intramuscular fat accumulation. After surgical reattachment, vascular structures with thicker walls were observed while the number of blood vessels remained unchanged. Our results support a potential role for muscle vascularization in tendon tear and repair, and could serve to advance the development of strategies to reduce intramuscular fat accumulation in rotator cuff tear.

\section{FUNDING}

This work was supported by the Workplace Safety and Insurance Board of Ontario (04031) and the Canadian Institutes of Health Research (MOP-81395). Author MMN was partially supported by the Hans K. Uhthoff FRCSC Graduate Fellowship. The authors, their immediate families, and any research foundations with which they are affiliated have not received any financial payments or other benefits from any commercial entity related to the subject of this article.

\section{ACKNOWLEDGEMENTS}

The study (ME-2479) was approved by the Animal Care Committee. We thank Julie Courchesne and Philippe Poitras for the surgeries, Mélanie Aubé Peterkin for intramuscular fat measurement, Dr. Ana Giassi for assistance with CD31 immunohistochemistry, Dr. Manijeh Daneschmand for imaging, and Dr. Tim Ramsay for statistical analysis.

4. Goutallier D, Postel J-M, Bernageau J, Lavau L, Voisin M-C. Fatty muscle degeneration in cuff ruptures. Pre- and postoperative evaluation by CT scan. Clin Orthop Relat Res [Internet] 1994; (304):78-83. Available from: http://content. whealth.com/linkback/openurl?sid = WKPTLP:landingpage\&an $=00003086-199407000-00014$

5. Oliva F, Osti L, Padulo J, Maffulli N. Epidemiology of the rotator cuff tears: a new incidence related to thyroid disease. Muscles Ligaments Tendons J 2014; 4(3):309-314.

6. Osti L, Buda M, Del Buono A. Fatty infiltration of the shoulder: Diagnosis and reversibility. Muscles Ligaments Tendons J 2013;3(4):351-354. 
7. Matsumoto F, Uhthoff HK, Trudel G, Loehr JF. Delayed tendon reattachment does not reverse atrophy and fat accumulation of the supraspinatus-an experimental study in rabbits. J Orthop Res [Internet] 2002; 20(2):357-363. Available from: http://www.ncbi.nlm.nih.gov/pubmed/11918317

8. Trudel G, Ryan S, Rakhra K, Uhthoff H. Extra- and intramuscular fat accumulation early after rabbit supraspinatus tendon division : Depiction with CT. Radiology 2010; 255(2):434-441.

9. Trudel G, Ryan SE, Rakhra K, Uhthoff HK. Muscle tissue atrophy, extramuscular and intramuscular fat accumulation, and fat gradient after delayed repair of the supraspinatus tendon: A comparative study in the rabbit. J Orthop Res 2012; 30(5):781-786.

10. Uhthoff H, Coletta E, Trudel G. Intramuscular fat accumulation and muscle atrophy in the absence of muscle retraction. Bone Joint Res 2014; 3(4):117-122.

11. Uhthoff H, Coletta E, Trudel G. Effect of timing of surgical SSP tendon repair on muscle alterations. J Orthop Res 2014; 32(11):1430-1435.

12. Rubino LJ, Stills HF, Sprott DC, Crosby LA. Fatty Infiltration of the Torn Rotator Cuff Worsens Over Time in a Rabbit Model. J Arthrosc Relat Surg 2007; 23(7):717-722.

13. Hausman GJ, Basu U, Du M, Fernyhough-Culver M, Dodson $\mathrm{M} \mathrm{V}$. Intermuscular and intramuscular adipose tissues: Bad vs. good adipose tissues. Adipocyte [Internet] 2014; 3(4): 242-255. Available from: http://www.tandfonline.com/doi/ abs/10.4161/adip.28546

14. Luan T, Liu X, Easley JT, Ravishankar B, Puttlitz C, Feeley BT. Muscle atrophy and fatty infiltration after an acute rotator cuff repair in a sheep model. Muscles Ligaments Tendons J 2015; 5(2):106-112.

15. Liu X, Ravishankar B, Ning A, Liu M, Kim HT, Feeley BT. Knocking-out matrix metalloproteinase-13 exacerbates rotator cuff muscle fatty infiltration. Muscles Ligaments Tendons J 2017; 7(2):202-207.

16. Hamrick MW, McGee-Lawrence ME, Frechette DM. Fatty Infiltration of Skeletal Muscle: Mechanisms and Comparisons with Bone Marrow Adiposity. Front Endocrinol (Lausanne) [Internet] 2016; 7:1-7. Available from: http://journal.frontiersin.org/Article/10.3389/fendo.2016.00069/abstract

17. Rowshan K, Hadley S, Pham K, Caiozzo V, Lee TQ, Gupta R. Development of Fatty Atrophy After Neurologic and Rotator Cuff Injuries in an Animal Model of Rotator Cuff Pathology. J Bone Jt Surgery-American Vol [Internet] 2010; 92(13):22702278. Available from: http://content.wkhealth.com/linkback/openurl?sid = WKPTLP:landingpage\&an =00004623201010060-00002

18. Christiaens V, Lijnen HR. Angiogenesis and development of adipose tissue. Mol Cell Endocrinol 2010; 318(1-2):2-9.

19. Frontini A, Corvera S, Cinti S. Origin of adipocyte prevursors from adipose vascular endothelium. In: Methods in Molecular Biology 2013; 65-81.

20. Determe D, Rongisres M, Kany J, Glasson JM, Bellumore Y, I $\mathrm{MM}$, et al. Anatomic study of the tendinous rotator cuff of the shoulder. Surg Radiol Anat 1996; 98:195-200.

21. Naidoo N, Lazarus L, De Gama BZ, Ajayi NO, Satyapal KS. Arterial Supply to the Rotator Cuff Muscles. Int J Morphol 2014; 32(321):136-140.

22. Korthuis RJ. Anatomy of Skeletal Muscle and Its Vascular Supply. In: Skeletal Muscle Circulation [Internet]. 2011:13-
8. Available from: http://www.ncbi.nlm.nih.gov/books/ NBK57140/

23. Padulo J, Oliva F, Frizziero A, Maffulli N. Muscles, ligaments and tendons journal - Basic principles and recommendations in clinical and field science research: 2016 update. Muscles Ligaments Tendons J 2016; 6(1):1-5.

24. Gerber C, Schneeberger AG, Perren SM, Nyffeler RW. Experimental rotator cuff repair. A preliminary study. J Bone Joint Surg Am 1999; 81(9):1281-1290.

25. Lohr JF, Uhthoff HK. The microvascular pattern of the supraspinatus tendon. Clin Orthop Relat Res [Internet] 1990; 254(254):35-38. Available from: https://www.ncbi.nlm.nih. gov/pubmed/2323147

26. Matthews JP. Vascular changes in flexor tendons after injury and repair : an experimental study. Injury 1977; 8:227-233.

27. Terrón VM, Muiños-López E, Granero-Moltó F, Prosper F, Pérez-Ruiz A, Pons-Villaneuva J. Musclar injuries after tendon rupture in the rotator cuff of animal models. Systematic review. Muscles, Ligaments and Tendons [Internet] 2018; 8(2):261-275. Available from: http://www.ncbi.nlm.nih.gov/ pubmed/26188754\%5Cnhttp://www.pubmedcentral.nih. gov/articlerender.fcgi? artid $=$ PMC4511038

28. Nourissat G, Berenbaum F, Duprez D. Tendon injury: From biology to tendon repair. Nat Rev Rheumatol [Internet] 2015; 11(4):223-233. Available from: http://dx.doi.org/10.1038/ nrrheum.2015.26

29. Cook JL, Malliaras P, De Luca J, Ptasznik R, Morris ME, Goldie P. Neovascularization and pain in abnormal patellar tendons of active jumping athletes. Clin J Sport Med 2004; 14(5):296-299.

30. Lakemeier S, Reichelt JJ, Patzer T, Fuchs-Winkelmann S, Paletta JR, Schofer MD. The association between retraction of the torn rotator cuff and increasing expression of hypoxia inducible factor $1 \alpha$ and vascular endothelial growth factor expression: an immunohistological study. BMC Musculoskelet Disord [Internet] 2010; 11(1):230. Available from: http://bmcmusculoskeletdisord.biomedcentral.com/articles/10.1186/1471-2474-11-230

31. Liu X, Ning AY, Chang NC, Kim H, Nissenson R, Wang L, et al. Investigating the cellular origin of rotator cuff muscle fatty infiltration and fibrosis after injury. Muscles Ligaments Tendons J 2016; 6(1):6-15.

32. Birbrair A, Zhang T, Wang Z-M, Messi ML, Enikolopov GN, Mintz A, et al. Role of pericytes in skeletal muscle regeneration and fat accumulation. Stem Cells Dev [Internet] 2013; 22(16):2298-2314. Available from: http://online.liebertpub. $\mathrm{com} / \mathrm{doi} / \mathrm{abs} / 10.1089 / \mathrm{scd} .2012 .0647$

33. Eilken HM, Diéguez-Hurtado R, Schmidt I, Nakayama M, Jeong H-W, Arf $\mathrm{H}$, et al. Pericytes regulate VEGF-induced endothelial sprouting through VEGFR1. Nat Commun [Internet] 2017; 8(1):1574. Available from: http://www.nature.com/ articles/s41467-017-01738-3

34. Medici D, Shore EM, Lounev VY, Kaplan FS, Kalluri R, Olsen BR. Conversion of vascular endothelial cells into multipotent stem-like cells. Nat Med 2010; 16(12):1400-1408.

35. Brioche T, Pagano AF, Py G, Chopard A. Muscle wasting and aging: Experimental models, fatty infiltrations, and prevention. Mol Aspects Med [Internet] 2015; 1-32. Available from: http://dx.doi.org/10.1016/j.mam.2016.04.006 
36. Yin H, Price F, Rudnicki MA. Satellite cells and the muscle stem cell niche. Physiol Rev [Internet] 2013; 93 (1):23-67. Available from: http://physrev.physiology.org/cgi/doi/10.1152/physrev.00043.2011

37. Harvey A, Montezano AC, Lopes RA, Rios F, Touyz RM. Vascular fibrosis in aging and hypertension: molecular mechanisms and clinical implications. Can J Cardiol [Internet] 2016; 32(5):659-668. Available from: http://dx.doi.org/10.1016/j. cjca.2016.02.070
38. Celermajer DS, Sorensen KE, Spiegelhalter DJ, Georgakopoulos D, Robinson J, Deanfield JE. Aging is associated with endothelial dysfunction in healthy men years before the age-related decline in women. J Am Coll Cardiol 1994; 24(2):471-476.

39. Jerusalem F, Rakusa M, Engel AG, MacDonald RD. Morphometric analysis of skeletal muscle capillary ultrastructure in inflammatory myopathies. J Neurol Sci 1974; 23(3):391-402.

40. Gregory CA, Prockop DJ, Spees JL. Non-hematopoietic bone marrow stem cells: Molecular control of expansion and differentiation. Exp Cell Res 2005; 306(2):330-335. 